\title{
Effect of gamma radiation on optical and electrical properties of tellurium dioxide thin films
}

\author{
T K MAITY and S L SHARMA* \\ Department of Physics \& Meteorology, Indian Institute of Technology, Kharagpur 721 302, India
}

MS received 27 February 2008; revised 22 May 2008

\begin{abstract}
Gamma radiation induced changes in the optical and electrical properties of tellurium dioxide $\left(\mathrm{TeO}_{2}\right)$ thin films, prepared by thermal evaporation, have been studied in detail. The optical characterization of the as-deposited thin films and that of the thin films exposed to various levels of gamma radiation dose clearly show that the optical bandgap decreases with increase in the gamma radiation dose up to a certain dose. At gamma radiation doses above this value, however, the optical bandgap has been found to increase. On the other hand, the current vs voltage plots for the as-deposited thin films and those for the thin films exposed to various levels of gamma radiation dose show that the current increases with the gamma radiation dose up to a certain dose and that the value of this particular dose depends upon the thickness of the film. The current has, however, been found to decrease with further increase in gamma radiation dose. The observed changes in both the optical and electrical properties indicate that $\mathrm{TeO}_{2}$ thin films can be used as the real time gamma radiation dosimeter up to a certain dose, a quantity that depends upon the thickness of the film.
\end{abstract}

Keywords. Tellurium dioxide; thin films; optical bandgap; gamma radiation dose; dosimeter.

\section{Introduction}

It is now a well known fact that the exposure of any solid material to ionizing radiations (such as X-rays, gamma rays, beta particles, alpha particles, fission fragments, etc) produces changes in the microstructural properties of the material, which in turn affects the optical and electrical properties (Ibrahim and Soliman 1998; Atanassova et al 2001; Clough 2001). Numerous efforts have recently been made to investigate the influence of gamma radiation on thin films and thin film structures of different metal oxides and polymers, in order to find out the suitability of using thin films and thin film structures of different metal oxides and polymers as gamma radiation dosimeters (Colby et al 2002; Arshak et al 2004, 2006; Arshak and Korostynska 2006). Naturally, a deep understanding of the physical properties of these thin films and thin film structures under the influence of gamma radiation is quite important from the viewpoint of design of novel and high sensitivity radiation dosimeters for gamma radiations.

The effect of gamma radiation on the microstructural, optical and electrical properties of tellurium dioxide $\left(\mathrm{TeO}_{2}\right)$ thin films and thin film structures has been the subject of several experimental investigations in the recent past (Arshak and Korostynska 2006; Arshak et al 2006; Kumar et al 2006). The $\mathrm{TeO}_{2}$ crystals exhibit re-

*Author for correspondence (shivji@phy.iitkgp.ernet.in) markable properties related to polarization and polarizability (dielectric, piezoelectric, etc), which are of great interest for the fundamental science and technology. The origin of such properties is related to the peculiarities of the electron distribution inside the coordination polyhedra (Mirgorodsky et al 2000).

Present work aims to study in detail the gamma radiation induced changes in both the optical and electrical properties of $\mathrm{TeO}_{2}$ thin films for a much wider range of gamma radiation doses than made here-to-fore. The work further aims to study the dose dependence of the current for $\mathrm{TeO}_{2}$ thin films of different thicknesses and then to determine the thickness dependence of the range of gamma radiation doses over which the current versus dose plot remains linear or near linear. This is quite important from the viewpoint of design of gamma radiation dosimeters from $\mathrm{TeO}_{2}$ thin films.

\section{Experimental}

Thin films of $\mathrm{TeO}_{2}$ were deposited on suitably prepared glass substrates by thermal evaporation in a vacuum of the order of $10^{-5}$ mbar. A disc-type ${ }^{60} \mathrm{Co}$ gamma radiation source was used to expose $\mathrm{TeO}_{2}$ thin films of different thicknesses to various dose levels at room temperature. For optical measurements, thin films of $\mathrm{TeO}_{2}$ of thickness of about $100 \mathrm{~nm}$ were deposited from a molybdenum boat on suitably prepared glass substrates. Thicker films were not suitable for the optical measurements due to high 
specular reflection. The optical absorption spectra for the as-deposited thin films as well as for the films exposed to different levels of gamma radiation dose were recorded using Perkin-Elmer Lambda 45 UV-Visible Spectrometer for the wavelengths in the range $270-770 \mathrm{~nm}$. For electrical measurement purposes, thin films having planar configuration as described earlier (Kumar et al 2006) were prepared. Two rectangular layers of aluminium, separated by $3 \mathrm{~mm}$ and each having thickness of about $150 \mathrm{~nm}$, were deposited on each of the suitably prepared glass substrates to act as electrical contacts. A tungsten filament in the form of a coil was used for the evaporation of aluminium. On the top of these aluminium contacts, thin films of $\mathrm{TeO}_{2}$ of thicknesses 300,450 and $600 \mathrm{~nm}$ were deposited from a molybdenum boat. For electrical measurements, thicker films were taken in order to have higher currents. The current vs applied voltage plots for the as-deposited thin films and for the thin films exposed to different levels of gamma radiation dose were recorded.

\section{Results and discussion}

As the optical properties of a material provide information about the electronic band structures, localized states and the nature (types) of optical transitions, the optical properties are very important for the understanding of the material. Therefore, the optical absorption spectra for the as-deposited thin films of $\mathrm{TeO}_{2}$ as well as for the $\mathrm{TeO}_{2}$ thin films exposed to different levels of gamma radiation dose were recorded and are shown in figure 1. These spectra were recorded for the thin films of thickness of about $100 \mathrm{~nm}$ and are quite similar to the spectra reported by Park and Woodward (2000) for the powdered samples of $\mathrm{TeO}_{2}$. Clearly, exposure to gamma radiation produces

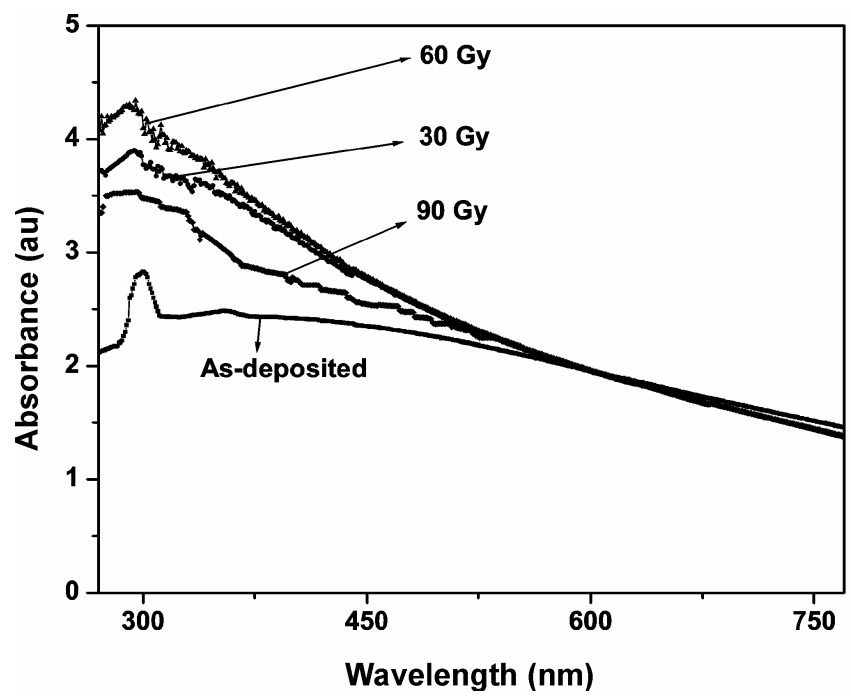

Figure 1. Typical optical absorption spectra for $\mathrm{TeO}_{2}$ thin films of thickness, $100 \mathrm{~nm}$, corresponding to different values of the gamma radiation dose considerable change in the shape of the optical absorption spectrum around the absorption edge. From an optical absorption curve, the values of the absorption coefficient, $\alpha_{v}$, for different values of the photon frequency, $v$, were calculated using the relationship

$$
\alpha_{v}=\frac{A_{v}}{t}
$$

where $A_{v}$ denotes the absorbance corresponding to the photon frequency, $v$ and $t$ the thickness of the thin film. The absorption coefficient near the band edge shows an exponential dependence on the photon energy and obeys the Urbach's empirical formula (Urbach 1953)

$$
\alpha_{v}=\alpha_{0} e^{(h v / \Delta E)}
$$

where $\alpha_{0}$ is a constant, $h$ the Planck's constant and $\Delta E$ the energy width of the band tails of localized states. The graphs of $\ln \alpha_{v}$ vs photon energy, $h v$, were plotted for the as-deposited thin films as well as for the films exposed to different levels of gamma radiation dose and the values of $\Delta E$ were calculated from the slopes of the straight-line portions of these plots. With $\Delta E_{0}$ as the energy width of the band tails of localized states for the as-deposited thin films, the variation of the normalized energy width, $\Delta E_{\mathrm{N}}\left(=\left(\Delta E-\Delta E_{0}\right) / \Delta E_{0}\right)$ of the band tails of localized states with the dose is shown in figure 2 . The energy width of the band tails of localized states has been found to increase with the gamma radiation dose up to a dose of about $60 \mathrm{~Gy}$. This increase in the energy width of the band tails of localized states can be attributed to the induced defects due to the exposure to gamma radiation. As the as-deposited $\mathrm{TeO}_{2}$ thin films are generally amorphous in nature, the atomic arrangement in terms of nearest neighbours departs only slightly from the ideal crystalline state. Further-

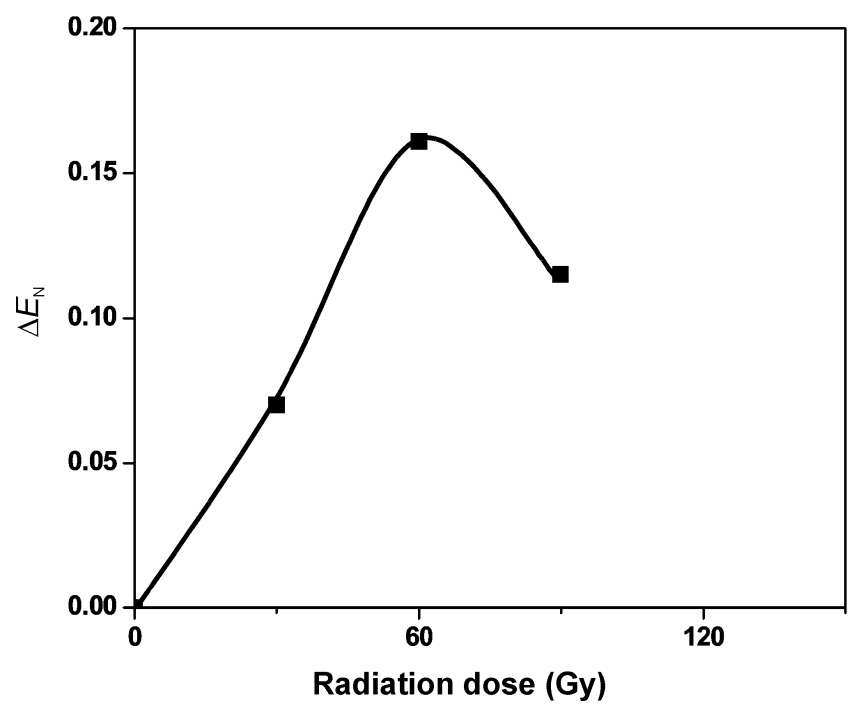

Figure 2. Variation of the normalized energy width of the band tails of localized states with the gamma radiation dose. 
more, since the amorphous thin films contain relatively rigid chemical bonds, the deviation from the crystalline state is small resulting in the requirement of excessive energy to distort this rigidity. This excessive energy leads to the formation of short-range order (Mott 1969) or creation of localized energy states in the normally forbidden energy gap. Whenever gamma radiation interacts with the thin film, induced defects will be formed and the density of the localized states increases resulting in increase in the energy width of the band tails of localized states. Arshak and Korostynska (2002) reported that the energy width of the band tails of localized states for the $\mathrm{TeO}_{2}$ thin films of thickness, $50 \mathrm{~nm}$, increased with the increase of radiation dose up to a value of $36 \mathrm{~Gy}$. They, however, did not

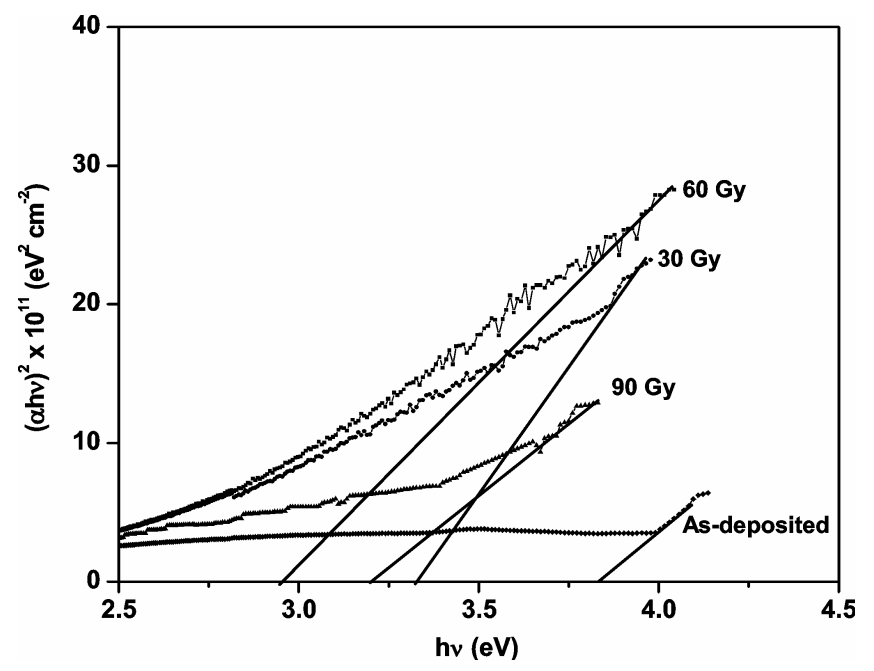

Figure 3. Plots of $\left(\alpha_{v} h v\right)^{2}$ vs $h v$ for $\mathrm{TeO}_{2}$ thin films of thickness, $100 \mathrm{~nm}$, corresponding to different values of the gamma radiation dose.

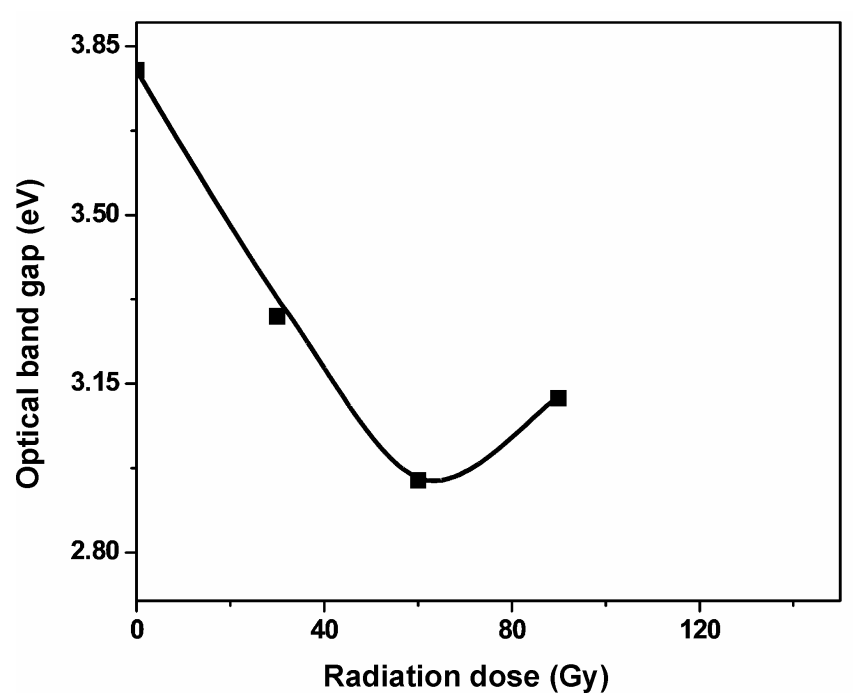

Figure 4. Variation of the optical bandgap with the gamma radiation dose. report for higher radiation doses. A comparison of the present results with those of Arshak and Korostynska (2002) clearly shows that the rate of increase of the energy width with radiation dose for thicker films is much lower than that for thinner films. Obviously, thin films are more sensitive than thick films.

The energy width of the band tails of localized states for the $\mathrm{TeO}_{2}$ thin films of thickness, $100 \mathrm{~nm}$, in the present study has, however, been found to decrease with further increase in the gamma radiation dose beyond $60 \mathrm{~Gy}$.

Figure 3 shows the plots of $\left(\alpha_{v} h v\right)^{2}$ vs $h v$ for the asdeposited thin films as well as for the thin films exposed to different levels of gamma radiation dose. The values of the optical bandgap, $E_{\text {opt }}$, for the as-deposited thin films and for the thin films exposed to different levels of gamma

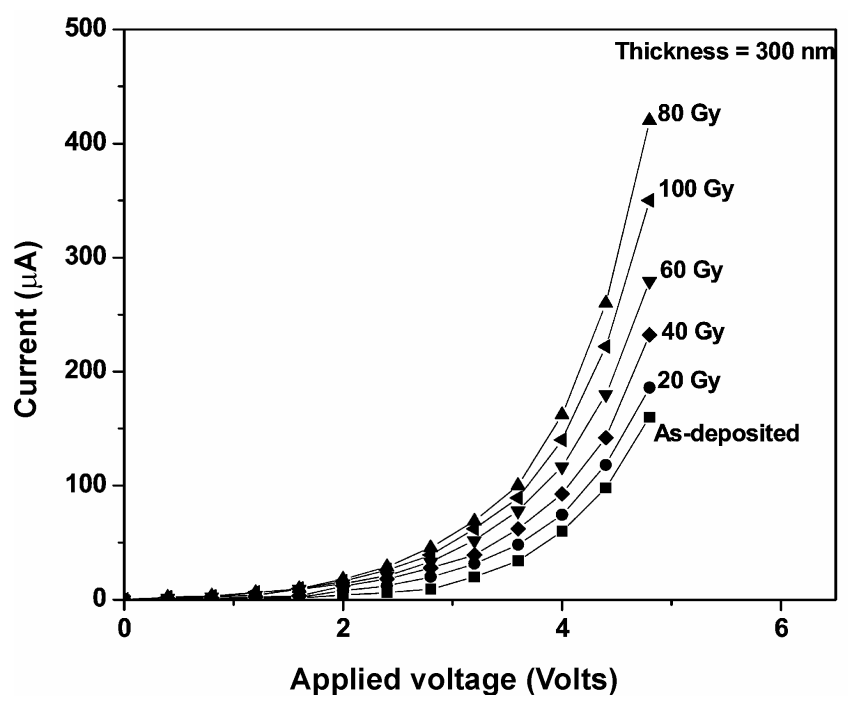

Figure 5. Current vs applied voltage plots for $\mathrm{TeO}_{2}$ thin films of thickness, $300 \mathrm{~nm}$, corresponding to different values of the gamma radiation dose.

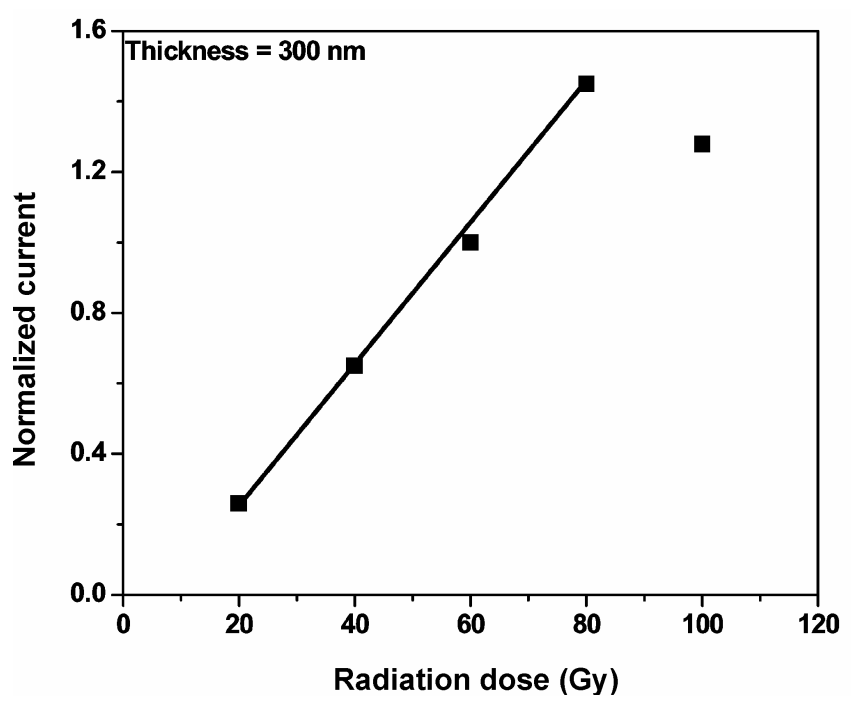

Figure 6. Typical variation of the normalized current with the gamma radiation dose for $\mathrm{TeO}_{2}$ thin films of thickness, $300 \mathrm{~nm}$, at an applied voltage of $3.5 \mathrm{~V}$. 
radiation dose were estimated using the Mott and Davis (1979) model for the direct allowed transition using the equation

$$
\alpha_{v} h v=B\left(h v-E_{\mathrm{opt}}\right)^{1 / 2},
$$

where $h v$ denotes the energy of the incident photon and $B$ a constant. The optical bandgap for the as-deposited thin films has been found to be about $3.8 \mathrm{eV}$, which matches reasonably well with the value reported in the literature (Arshak and Korostynska 2006). The difference may be attributed to the different conditions under which thin films were prepared. The variation of the optical bandgap with the radiation dose is shown in figure 4 . Clearly, the optical bandgap decreases from $3.80 \mathrm{eV}$ to $2.95 \mathrm{eV}$ for the gamma radiation dose of $60 \mathrm{~Gy}$. This decrease in the optical bandgap is basically due to the increase in the energy width of the band tails of localized states. During gamma irradiation, the defects are created within the thin film. At the same time, the defects also get annihilated even under the normal room temperature conditions (Deng et al 1999). This creation and annihilation of defects coexist together and at higher doses of gamma radiation, the number of defects created due to irradiation becomes much more than the number of defects annihilated. Arshak and Korostynska (2002) reported that the optical bandgap for the $\mathrm{TeO}_{2}$ thin films of thickness, $50 \mathrm{~nm}$, decreased from $3.75 \mathrm{eV}$ for as-deposited films to $3.45 \mathrm{eV}$ for the films exposed to gamma radiation dose of $36 \mathrm{~Gy}$. They, however, did not report for higher radiation doses. A comparison of the present results with those of Arshak and Korostynska (2002) clearly indicates the weak dependence of the rate of decrease of the optical bandgap with radiation dose on the film thickness.

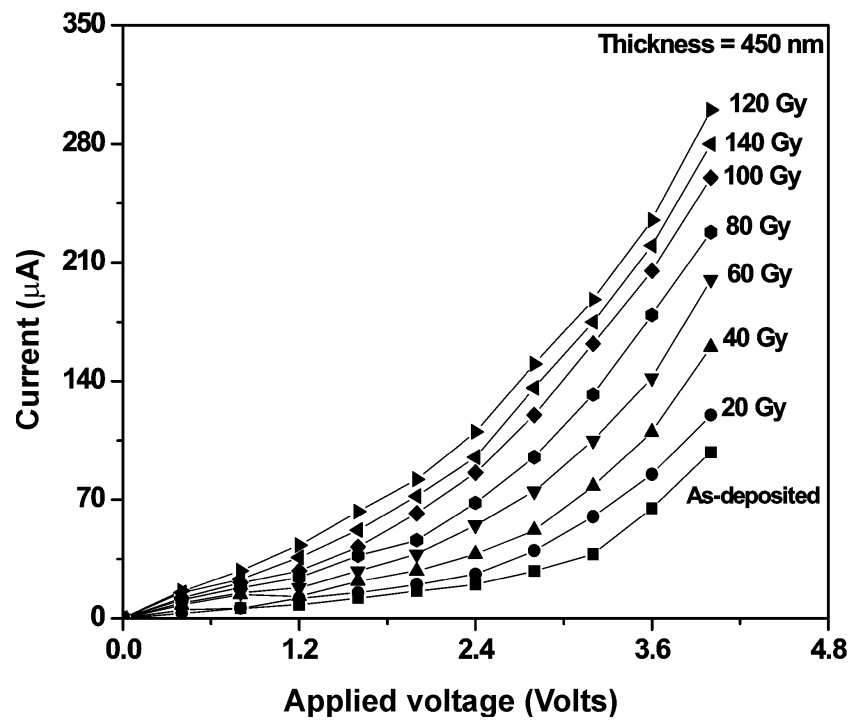

Figure 7. Current vs applied voltage plots for $\mathrm{TeO}_{2}$ thin films of thickness, $450 \mathrm{~nm}$, corresponding to different values of the gamma radiation dose
The optical bandgap for the $\mathrm{TeO}_{2}$ thin films of thickness, $100 \mathrm{~nm}$, in the present work has, however, been found to increase with further increase in the gamma radiation dose beyond $60 \mathrm{~Gy}$.

The behaviour of the optical properties of $\mathrm{TeO}_{2}$ thin films, described above, is quite similar to the doseresponse of most materials used in thermoluminescence dosimetry (Horowitz 2001). These materials usually show a linear, then super-linear, followed by saturating response and further increase in dose results in the high structural disorder. The high structural disorder at very high radiation doses reduces the sensitivity of material parameters to further exposure of radiation. The increase in the optical bandgap for the $\mathrm{TeO}_{2}$ thin films of thickness, $100 \mathrm{~nm}$, for the gamma radiation doses beyond $60 \mathrm{~Gy}$ may be attributed to a similar high structural disorder at very high radiation doses. Arshak et al (2006) have also reported a similar observation for the thin films of the mixture of $\mathrm{MnO} / \mathrm{TeO}_{2}$.

Figure 5 shows the current vs applied voltage plots for the $\mathrm{TeO}_{2}$ thin films of thickness, $300 \mathrm{~nm}$, recorded for the as-deposited thin films as well as for the thin films exposed to different levels of gamma radiation dose. The variation of the normalized current, $I_{\mathrm{N}}$ (defined below), with the dose up to a gamma radiation dose of $100 \mathrm{~Gy}$ for the applied voltage of $3.5 \mathrm{~V}$ is shown in figure 6 .

$$
I_{N}=\frac{I-I_{0}}{I_{0}}
$$

where $I$ denotes the current for the thin films subjected to a particular value of the gamma radiation dose and $I_{0}$ the current for the as-deposited thin films. Clearly, the cur-

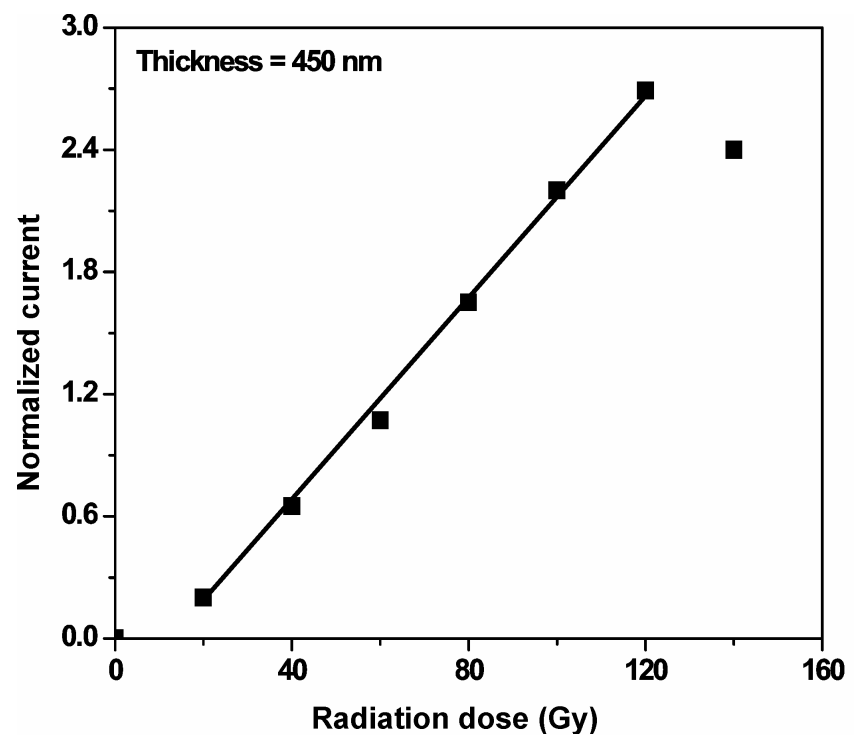

Figure 8. Typical variation of the normalized current with the gamma radiation dose for $\mathrm{TeO}_{2}$ thin films of thickness, $450 \mathrm{~nm}$, at an applied voltage of $3.5 \mathrm{~V}$. 
rent increases quite linearly with the dose up to the gamma radiation dose of $80 \mathrm{~Gy}$. The current has, however, been observed to decrease beyond this dose for the $\mathrm{TeO}_{2}$ thin films of thickness, $300 \mathrm{~nm}$.

The current vs applied voltage plots for the $\mathrm{TeO}_{2}$ thin films of thickness, $450 \mathrm{~nm}$, recorded for the as-deposited thin films as well as for the thin films exposed to different levels of gamma radiation dose are shown in figure 7 . Figure 8 shows the variation of the normalized current, $I_{\mathrm{N}}$, with the dose up to a gamma radiation dose of $140 \mathrm{~Gy}$ for the applied voltage of $3.5 \mathrm{~V}$. Clearly, the current increases near linearly with the dose up to the gamma radiation dose of $120 \mathrm{~Gy}$. The current has, however, been observed to decrease beyond this dose for the $\mathrm{TeO}_{2}$ thin films of thickness, $450 \mathrm{~nm}$.

Figure 9 shows the current vs applied voltage plots for the $\mathrm{TeO}_{2}$ thin films of thickness, $600 \mathrm{~nm}$, recorded for the as-deposited thin films as well as for the thin films exposed to different levels of gamma radiation dose. The variation of the normalized current, $I_{\mathrm{N}}$, with the dose up to a gamma radiation dose of $200 \mathrm{~Gy}$ for the applied voltage of $3.5 \mathrm{~V}$ is shown in figure 10 . Clearly, the current increases near linearly with the dose up to the gamma radiation dose of $160 \mathrm{~Gy}$. Subsequently, however, the current has been observed to decrease.

Present studies have clearly shown that the gamma radiation dose up to which the current keeps increasing near linearly depends upon the thickness of the $\mathrm{TeO}_{2}$ thin films and that it is $80 \mathrm{~Gy}$ for the thin films of thickness $300 \mathrm{~nm}, 120 \mathrm{~Gy}$ for the thin films of thickness, $450 \mathrm{~nm}$ and $160 \mathrm{~Gy}$ for the thin films of thickness, $600 \mathrm{~nm}$. For the $\mathrm{TeO}_{2}$ thin films of thicknesses, 300,450 and $600 \mathrm{~nm}$, however, the current has been observed to decrease beyond the gamma radiation doses of 80,120 and $160 \mathrm{~Gy}$,

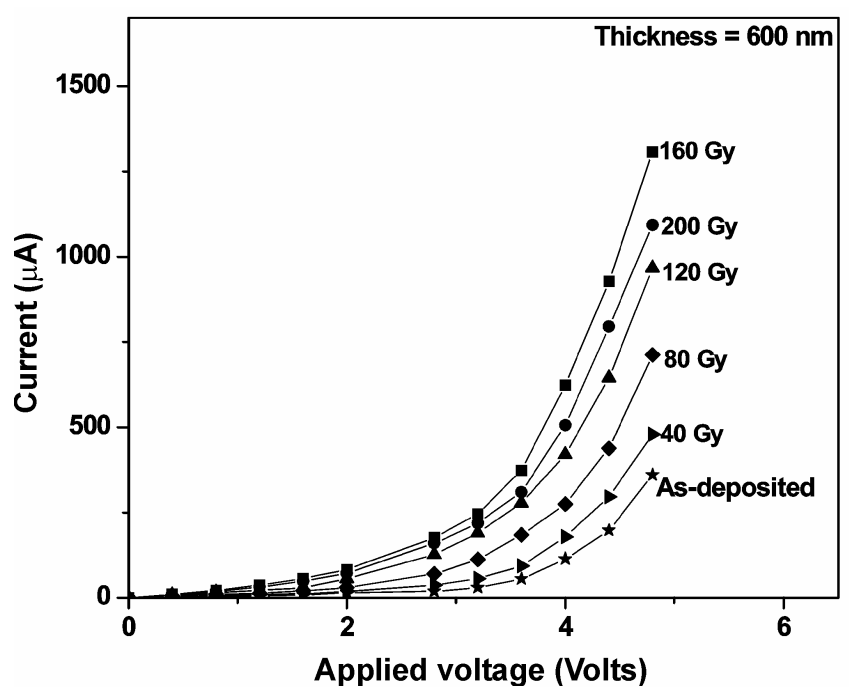

Figure 9. Current vs applied voltage plots for $\mathrm{TeO}_{2}$ thin films of thickness, $600 \mathrm{~nm}$, corresponding to different values of the gamma radiation dose. respectively. Recently, Arshak et al (2004) have also reported that the sensitivity of the thin films for low radiation doses is much higher than that of the thick films and that the thick films sustain up to much higher radiation doses than the thin films.

The current for the $\mathrm{TeO}_{2}$ thin films has been observed to increase near linearly with the dose up to a certain gamma radiation dose (critical dose) and the value of this dose is higher for the thicker films. This increase in the current may be attributed to the healing effect (Zaykin and Aliyev 2002). During the process of film deposition, some intrinsic defects are always formed. The interaction of gamma radiation induces defects during its passage through the thin film resulting into disorder in the microstructure of the film. At small doses, these thin films have fine homogeneous grain structure without any big pores and the number of defects (induced plus residual intrinsic) is smaller than the number of intrinsic defects due to the recombination of defects. The recombination of defects reduces the resistivity of thin film, giving rise to an increase in the current. The critical dose dependence on the film thickness can also be understood in terms of the healing effect (Zaykin and Aliyev 2002). Accordingly, thicker films require much higher radiation doses for healing in comparison to thinner films.

The near linear increase of current with the gamma radiation dose can also be understood in terms of a simple charge transfer model (Arshak and Korostynska 2006), according to which the Fermi level shifts upward in the exposed thin films as compared to that in the as-deposited thin films which then results in lowering of the optical bandgap. In turn, the filling of the hole-states causes the current to increase with the increase in the gamma radia-

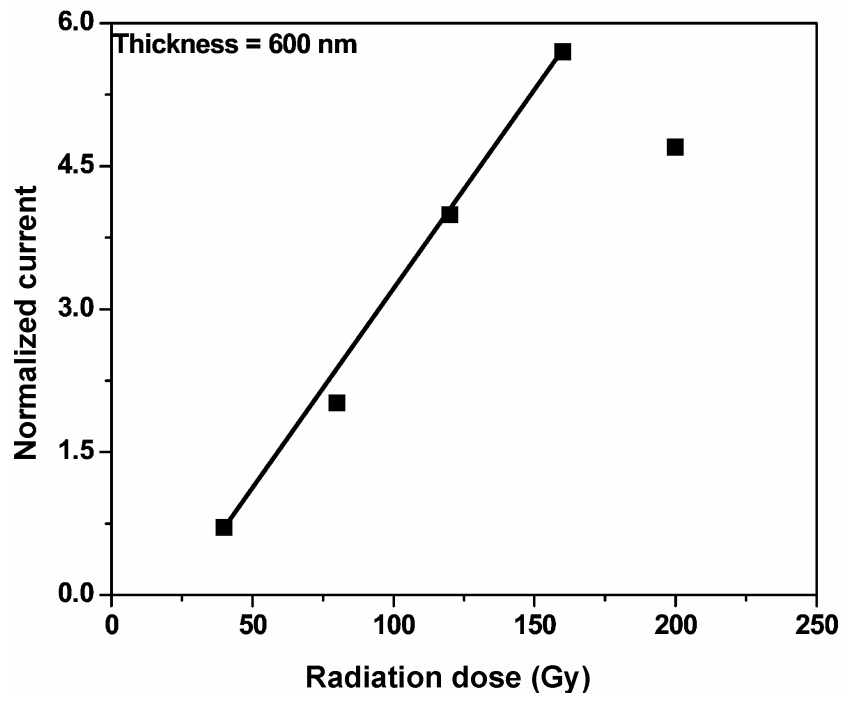

Figure 10. Typical variation of the normalized current with the gamma radiation dose for $\mathrm{TeO}_{2}$ thin films of thickness, $600 \mathrm{~nm}$, at an applied voltage of $3.5 \mathrm{~V}$. 
tion dose up to a certain dose. The current is, however, observed to decrease with the increase in the radiation dose above a certain dose level which may be partly attributed to the increase in the optical bandgap (see figure 4).

The near linear variation of the current with the gamma radiation dose up to a certain critical dose (a quantity dependent upon the thickness of the film) can be considered as a working region for gamma radiation dosimetry.

\section{Conclusions}

The optical absorption studies on thermally evaporated $\mathrm{TeO}_{2}$ thin films, exposed to various levels of gamma radiation dose, clearly show that the optical bandgap decreases with the increase in gamma radiation dose up to a certain dose and that the optical bandgap increases with further increase in the dose. On the other hand, the current-voltage characteristics show that the current increases near linearly with the gamma radiation dose up to a certain critical dose (a quantity dependent upon the thickness of the film) and that the current decreases for the gamma radiation doses above this value. The near linear variation of the current with the radiation dose clearly indicates that the $\mathrm{TeO}_{2}$ thin films have high potential for their use in the real time gamma dosimetry.

\section{References}

Arshak K and Korostynska O 2002 Sensors 2347

Arshak K and Korostynska O 2006 Mater. Sci. Eng. B133 1

Arshak K, Arshak A, Zleetni S and Korostynska O 2004 IEEE Trans. Nucl. Sci. NS-51 2250

Arshak K, Korostynska O, Molly J and Harris J 2006 IEEE Sensors J. 6656

Atanassova E, Pasksleva A, Konakova R, Spassov D and Mitin V F 2001 Microelectronics J. 32553

Clough R L 2001 Nucl. Instrum. Meth. B185 8

Colby E, Lum G, Plettner T and Spencer J 2002 IEEE Trans. Nucl. Sci. NS-49 2857

Deng Q, Yin Z and Zhu R 1999 Nucl. Instrum. Meth. A438 415

Horowitz Y S 2001 Nucl. Instrum. Meth. B184 68

Ibrahim A M and Soliman L I 1998 Rad. Phys. \& Chem. 53 469

Kumar G A, Maity T K, Kumar A and Sharma S L 2006 Int. conf. on advances in materials and materials processing (Kharagpur: IIT) p. 913

Mirgorodsky A P, Merle-Mejean T, Champarnaud J C, Thomas P and Frit B 2000 J. Phys. \& Chem. Solids 61501

Mott N F 1969 Philos. Mag. 1919

Mott N F and Davis E 1979 Electronic process in noncrystalline materials (UK: Clarendon Press)

Park J H and Woodward P M 2000 Int. J. Inorg. Mater. 2153

Urbach F 1953 Phys. Rev. 921324

Zaykin Y A and Aliyev B A 2002 Rad. Phys. \& Chem. 63227 